\title{
Triple-coincidence experiment to study the correlated electron emission in the collision-induced double electron loss of $\mathrm{He}^{-}$
}

\author{
L. Sarkadi $\odot^{*}$ and A. Orbán \\ Institute for Nuclear Research of the Hungarian Academy of Sciences (MTA Atomki), P.O. Box 51, H-4001 Debrecen, Hungary
}

(Received 29 July 2019; published 25 November 2019)

\begin{abstract}
The double electron loss (DEL) from $\mathrm{He}^{-}$has been investigated in collisions with $\mathrm{He}$ atoms at $300-\mathrm{keV}$ impact energy and at electron energies that satisfy the cusp condition; i.e., the corresponding electron velocities match the projectile's velocity. In a time-of-flight experiment the energies of both electrons have been determined by detecting triple coincidences between the two electrons and the outgoing $\mathrm{He}^{+}$ion. The coincidence yield shows a peak as a function of the electron energies at the expected cusp position, providing evidence for the existence of the two-electron cusp [L. Sarkadi and A. Orbán, Phys. Rev. Lett. 100, 133201 (2008)] in DEL. The observed energy distribution of the electron pairs is compared with the results of four-body classical trajectory Monte Carlo calculations carried out applying the soft-Coulomb potential approximation for the inclusion of the electron-electron interaction.
\end{abstract}

DOI: 10.1103/PhysRevA.100.052708

\section{INTRODUCTION}

The behavior of the scattering processes near a reaction energy threshold has been widely investigated in different fields of physics since the pioneering work of Wigner [1] (in atomic and molecular physics see, e.g., the reviews [2,3]). The continuing interest is mainly explained by the high degree of universality of the threshold laws governing the processes: The energy dependence of the cross section in the neighborhood of the threshold energy is determined only by the type of the interaction (long or short range, attractive or repulsive), and it is not sensitive to the details of the collision system. The universality can be traced back to the large de Broglie wavelength of the slow collision fragments compared to the characteristic length of the interaction. The latter property is attractive from a theoretical point of view, because it allows the application of analytical methods in the description of the collision dynamics.

In atomic physics the most well-known threshold process is the near-threshold single ionization of an atom by electron impact (or photo-double ionization of an atom). Applying classical mechanics, Wannier [4] showed that the motion of the two low-energy electrons emerging from the collision is highly correlated. Exactly at the threshold the electrons move symmetrically in opposite directions; above the threshold their angular correlation is expressed by a Gaussian centered at $\theta_{12}=\pi$ with a width that depends on the excess energy of the collision as $\sim E^{1 / 4}$. Furthermore, Wannier derived an $E^{1.127}$ dependence of the ionization cross section on the excess energy, and he found a uniform energy sharing between the two outgoing electrons. We emphasize that the dependence of the angular correlation and the cross section on the excess energy is universal for all atomic species. The predictions of

\footnotetext{
*sarkadil@atomki.hu
}

the Wannier theory have been proved experimentally (see, e.g., [5-11]) and confirmed by a large number of further theoretical works (see, e.g., [12-19]) for both $(e, 2 e)$ and $(\gamma, 2 e)$.

In the field of energetic ion-atom collisions the study of the so-called electron cusp has been proved to be an efficient tool to check the validity of the threshold laws. The cusp is a peak (a singularity) appearing in the energy spectrum of the electrons observed in the forward direction. The peak is formed by the electrons ejected with velocities approximately equal to that of the bombarding ion, i.e., they fly with small velocities relative to the projectile. The cusp is regarded as a threshold phenomenon, because the final relative electronprojectile energy is just above the ionization limit. A negative relative energy would produce the population of the bound states of the projectile ion characterized by a large principal quantum number (Rydberg states). The study of the cusp with different projectiles makes it possible to obtain information about the threshold laws for various types of interactions (Coulomb, dipolar, short range; see, e.g., [20-26]). In the special case of the attractive Coulomb interaction the Wigner laws predict a nonzero cross section at the threshold (see, e.g., [2]), and this leads to a cusp, as we will show in Sec. III B.

On the basis of the universality of the threshold processes one may assume that the Wannier-type correlated twoelectron states can be formed also in ion-atom collisions. Final states consisting of two low-energy electrons in the field of a positive ion are expected to be formed via simultaneous emission of two electrons in forward direction with the same velocity as that of the projectile. While single electron emission leads to a cusp in the energy spectrum of the electrons, it is an interesting question whether the cusp exists also for double electron emission. In [27] we reported about an experiment on the existence of the two-electron cusp. In the experiment the energies of the two electrons resulting from the simultaneous target and projectile ionization in 
$100-\mathrm{keV} \mathrm{He}^{0}+\mathrm{He}$ collisions were measured by detecting triple coincidence between the electrons and the outgoing $\mathrm{He}^{+}$ ion. The coincidence yield was found to peak at the expected cusp position as a function of the electron energies, proving the existence of the two-electron cusp. Furthermore, a strong correlation between the electron energies in the vicinity of the cusp was observed which was traced back to an angular correlation of $180^{\circ}$ in the projectile frame.

In [27] we presented the results of a Monte Carlo simulation based on Wannier's theory. In the simulation we randomly created low-energy electron pairs in the projectile-centered reference frame. The energy- and angular distribution of the electrons were taken as predicted by Wannier's theory. The paths of the electrons then were tracked until they reached the detectors. The simulation resulted in an energy correlation in agreement with the observation, thus providing a strong indication for the formation of the correlated two-electron Wannier states in ion-atom collisions.

In subsequent theoretical works on the two-electron cusp $[19,28]$ attempts were made to reproduce the observed energy correlation. In these works a simpler collision system was considered, namely, the double ionization (DI) of $\mathrm{He}$ by $\mathrm{He}^{2+}$, instead of the complicated mutual ionization of the target and projectile in $\mathrm{He}^{0}+\mathrm{He}$ collision. In the former collision DI leads to low-energy electrons relative to the projectile by a special process, known as electron capture into the continuum (ECC). The calculations were made in two models. Both approaches treated the electron emission in the independent particle model using the continuum distorted wave method, but applied different correction methods for the inclusion of the electron correlation. Gulyás et al. [28] proposed a correction procedure based on the Coulomb density of states approximation. In their model the electron-electron interaction was taken into account in the final continuum state of the electrons by introducing a dynamically screened effective charge in the Coulomb normalization factor of the wave function. Barrachina et al. [19] considered the quantum mechanical version of Wannier's theory, and derived a correlation factor within the WKB approximation. The predictions of both models were found only in qualitative agreement with the experiment.

In the present paper we continued the experimental investigation of the two-electron cusp. Now we considered a collision system in which the two electrons originate from the same collision partner, facilitating thereby the theoretical description. One possible choice is the double ECC in $\mathrm{He}^{2+}$ + He collision considered in the above theoretical models, as well as the double electron loss (DEL) of $\mathrm{He}^{0}$ in collision with He. However, test experiments for these systems resulted in very low triple-coincidence yields, which can be explained by the large binding energies of the electrons in He. A reasonable coincidence yield is expected for an atom (ion) having two loosely bound electrons. A good choice from this point of view is the $\mathrm{He}^{-}$ion that exists only in the core-excited $(1 s 2 s 2 p){ }^{4} P^{o}$ state. Considering that the energy of this state is -2.178 a.u. [29], the total binding energy of the outer $2 s$ and $2 p$ electron with respect to the $1 s$ core state is only -0.178 a.u. $(-4.84 \mathrm{eV})$. The $(1 s 2 s 2 p)^{4} P^{o}$ state is metastable. However, its lifetime is relatively long, $359 \mu \mathrm{s}$ [30], which enables the use of $\mathrm{He}^{-}$in collision experiments (see, e.g., $[23,31,32]$ ).
Based on the above arguments, we chose the double electron loss of $\mathrm{He}^{-}$induced by collisions with $\mathrm{He}$ atoms for the present investigation of the two-electron cusp. At first glance, this five-electron system seems to be too complex; the detection of a triple coincidence of an electron pair with the outgoing $\mathrm{He}^{+}$ion does not identify unambiguously the DEL of $\mathrm{He}^{-}$. However, we may assume that at the chosen impact energy $(300 \mathrm{keV})$ the ionization of the loosely bound $2 s$ and $2 p$ electrons is dominant in the collision; the strongly bound electrons in the He target and the $1 s$ core electron of the $\mathrm{He}^{-}$ projectile practically remain intact.

The organization of the paper is as follows. In Sec. II, we summarize the applied experimental procedure. In Sec. III, we present a four-body classical trajectory Monte Carlo (CTMC) model worked out for the interpretation of the results of the present experiment, as well as a Monte Carlo simulation by which the energy correlation pattern of the electron pairs can be generated according to Wannier's theory. In Sec. IV, we compare the obtained experimental data with the results of the CTMC calculations. Furthermore, using the Monte Carlo simulation we analyze how far the experimental data reflect the Wannier-type electron correlation.

\section{EXPERIMENTAL METHOD}

The measurements were performed at the 1.5-MV Van de Graaff accelerator of Atomki. The experimental setup was an upgraded version [25] of the arrangement that had been applied in the previous investigation of the two-electron cusp [27]. The $300-\mathrm{keV} \mathrm{He}^{-}$beam was produced from the $\mathrm{He}^{+}$ beam of the accelerator via charge exchange with the residual gas of the beam channel. The negative ion component was selected from the original beam with a four-stage electrostatic charge-state selector [20]. The final beam of diameter $0.75 \mathrm{~mm}$, containing about $10^{6}$ ions $/ \mathrm{s}$, was formed by a collimator of length $45 \mathrm{~cm}$.

The electrons ejected in forward direction from the interaction of the ion beam and an effusive He gas target are reflected by an electrostatic mirror [33] into backward angles $\left(\approx 160^{\circ}\right)$, while the projectile passes through the mirror. The reflected electrons fly through a drift tube and are then detected by two channel electron multipliers (CEMs). The outgoing projectile ion is charge-state analyzed by means of an electrostatic deflector and detected by an ion detector [34]. The energies of the electrons are determined from the time of flight (TOF) of the electrons, obtained as the difference between the arrival time of the electrons and that of the charge-state selected $\mathrm{He}^{+}$ ion. The data acquisition was made in list mode applying a time-to-digital converter developed in Atomki. The time resolution was $5 \mathrm{~ns}$. It was determined practically by the electron and ion detection (including electronics); the contribution of the time dispersion in the mirror was negligible.

The base pressure in the collision chamber was $1.5 \times$ $10^{-6}$ mbar. It increased to $6 \times 10^{-6}$ mbar when the gas target was turned on. The single-collision condition was checked by measuring the triple-coincidence yield as a function of the target gas pressure. We found a linear dependence. Typical rates of the ions, electrons, and double and triple coincidences were as follows: $\mathrm{He}^{+}$ions, $20 \mathrm{kHz}$; electrons, $0.5 \mathrm{kHz}$; electron-ion coincidences, $20 \mathrm{~Hz}$; triple coincidences, $0.03 \mathrm{~Hz}$. 
The upgrade of the setup involved the replacement of the drift tube by a three times longer one, which substantially improved the energy and angular resolution. Furthermore, in the tube we built in a five-element electrostatics lens for focusing, accelerating, or decelerating the electrons. One of the lens elements was constructed as a four-electrode steering system to correct the electron trajectories [35]. An axial magnetic field serving to guide the electrons towards the detectors can also be produced by a solenoid coil mounted on the tube.

As we emphasized in [33], one of the most important requirements in the design of TOF was the minimized secondary electron production in the parts of the spectrometer. This was achieved mainly by constructing the mirror without a grid. However, secondary electrons may also be produced on the surface of the input apertures of the two CEMs. The CEMs are parts of a detector array consisting of four CEMs (Sjuts Optotechnik, type KBLA 510-4). The apertures have rectangular shape; their size is $5 \times 10 \mathrm{~mm}^{2}$. The neighboring apertures are separated by $1 \mathrm{~mm}$, therefore electrons hitting the surface of the separating area of $1 \times 10 \mathrm{~mm}^{2}$ may eject a secondary electron. To suppress the false electron-electron coincidences due to the secondary electron production, as a further upgrade of the setup we mounted on the detector array an electrode system consisting of two additional apertures of the same shape as that of the input apertures. Furthermore, to reduce the effective surface of the secondary electron production, we applied a Soller collimator built up from five copper plates of $0.1-\mathrm{mm}$ thickness. By applying suitable voltages on the electrodes, we completely reduced the effect of the secondary electrons.

In the present triple-coincidence experiment the electron energy covered a range between 28 and $56 \mathrm{eV}$. To achieve a reasonable energy resolution, the electrons were decelerated by $-20 \mathrm{~V}$. In the considered range the relative energy resolution of TOF changed almost linearly from 0.5 to $2.6 \%$, i.e., the energy resolution varied from 0.15 to $1.5 \mathrm{eV}$.

For the energy calibration of TOF we determined the relationship between the energy and time of flight of the electrons following their path from the target to the detectors. For tracking the trajectories in the mirror and in the lens we used the SIMION three-dimensional (3D) ion optics computer program (version 8.0). The calibration was checked by measuring the energy position of the single electron cusp for different collision systems at several impact energies in the range between 150 and $300 \mathrm{keV}$. The measured positions agreed well with the expected values, $(m / M) E_{\mathrm{p}}$, where $m$ is the mass of the electron, and $M$ and $E_{\mathrm{p}}$ are the mass and the energy of the projectile, respectively. The energy dispersion of the ion beam and its energy loss due to the ionization were negligible $(0.5$ and $0.015 \%$, respectively) compared to the relative energy resolution of TOF, which was $1.4 \%$ at the cusp position.

As far as the angular resolution is concerned, it was limited by the low triple-coincidence yield, i.e., we had to operate TOF with a modest resolution. At the same time, a pronounced cusp can only be obtained with a relatively good angular resolution. This means that we optimized TOF in a way to achieve an acceptable (narrow enough) cusp shape and a reasonable coincidence yield. The optimization was made by varying both the electrode voltages of the lens and the magnetic field in the drift tube. We note that at a given value of deceleration the optimization of the angular resolution had only a small effect on the energy resolution, because the variation of the voltages of the lens electrodes caused only small changes in the time of flight of the electrons.

Due to the complexity of TOF, we could not determine the acceptance (half) angle of the electron detection purely from the geometry and focusing properties of the system. Considering that the knowledge of this parameter is unavoidable in the theoretical analysis of the experimental results, we estimated it by performing a separate experiment and theoretical calculations. In the experiment we measured the single electron ECC cusp in $300-\mathrm{keV} \mathrm{He}^{2+}+\mathrm{He}$ collisions by coincident detection of the electrons with the outgoing $\mathrm{He}^{2+}$ ions under the same conditions used in the triple-coincidence experiment. We compared the measured cusp shape with the result of CTMC calculations. We used a three-body version of CTMC, applying a screened Coulomb potential for the target core. This model proved to be efficient in the description of the electron emission in ion-atom collisions, including the cusp (see, e.g., [36]). We calculated the cusp using different values for the acceptance angle. We found good agreement between the calculated and measured shape at $\Delta \vartheta_{1 / 2} \approx 1^{\circ}$.

In the same way, we determined the energy-dependent electron detection efficiency (transmission of TOF and detection efficiency of the channeltrons for the two electron branches) by taking the ratio of measured and calculated electron energy spectra. We measured only relative cross sections for the two-electron cusp, therefore we determined only relative efficiency. For this purpose we considered the $150-\mathrm{keV} \mathrm{H}^{0}+\mathrm{He}$ collision, in which the electrons are dominantly produced by the ionization of the projectile (single electron loss, SEL). The process was identified by detecting the electrons in coincidence with the outgoing protons. The impact energy was chosen in a way to avoid the cusp region. In the vicinity of the cusp the spectrum changes rapidly, and the determination of the efficiency is uncertain. For $150-\mathrm{keV}$ $\mathrm{H}^{0}$ impact the cusp appears at $81.6 \mathrm{eV}$; i.e., the energy range of the triple-coincidence experiment $(28-56 \mathrm{eV})$ is located well below the cusp maximum. For the theoretical derivation of the spectrum we used the above CTMC model. For the description of SEL we considered the inverse reaction, i.e., the ionization of the hydrogen target atom by the completely screened helium atom projectile. Since the coincidence condition did not exclude the simultaneous ionization of both the target and projectile, the contribution of this process was also included in the CTMC calculations. As a cross checking, we repeated the procedure at $300-\mathrm{keV}$ impact energy. We obtained a reasonable agreement, and the final efficiency was taken as the average of the $150-$ and $300-\mathrm{keV}$ results.

The contribution of the accidental coincidence events to the true triple-coincidence events was not negligible, and we determined it as follows. The accidental coincidence events have four sources, as it is seen in the 3D representation of the distribution of the detected triple coincidences as a function of the time of flight of the two electrons, $\Delta t_{1}$ and $\Delta t_{2}$ [see Fig. 1(a)]: (i) true $\mathrm{He}^{+}-e_{1}$ correlated events in random coincidence with $e_{2}$ producing the ridge at a constant $\Delta t_{1}$ value; (ii) true $\mathrm{He}^{+}-e_{2}$ correlated events in random coincidence with $e_{1}$ producing the ridge at a constant $\Delta t_{2}$ value; 


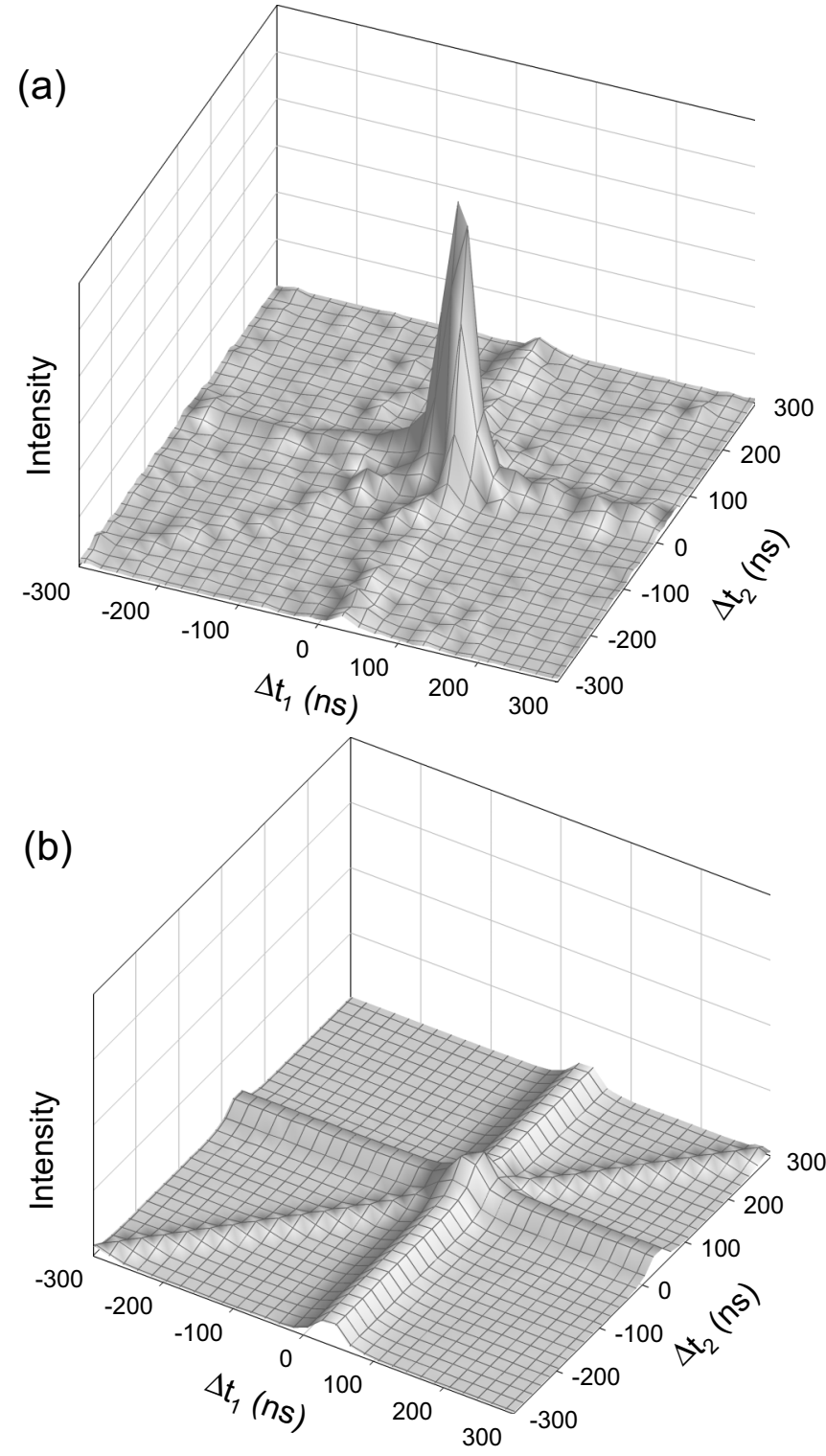

FIG. 1. (a) The distribution of the measured triple-coincidence events as a function of the time of flight of the two electrons emitted by DEL of $\mathrm{He}^{-}$. (b) The contribution of the accidental coincidences reconstructed from the measured ion-electron and electron-electron double-coincidence distributions. The fully random coincidences between the three particles are accounted for by a uniform distribution.

(iii) true $e_{1}-e_{2}$ correlated events in random coincidence with $\mathrm{He}^{+}$producing the ridge at $\Delta t_{1} \approx \Delta t_{2}$; and (iv) fully random coincidences between the three particles producing a uniform distribution. The shapes of the distributions of the "semirandom" events can be deduced from the corresponding double-coincidence time spectra. The latter are known very accurately due to the good counting statistics accumulated during the long-time triple-coincidence measurements. The total accidental "background" reconstructed from the measured ion-electron and electron-electron double-coincidence distributions is seen in Fig. 1(b). The contribution of ionelectron-electron random coincidences is accounted for by a uniform distribution. The weights of the different components of the accidental background were determined by a suitable normalization of the reconstructed distribution to the measured one. This was done by selecting areas in the $\Delta t_{1}-\Delta t_{2}$ plane, where only the regarded component is expected to contribute to the random coincidences.

As a result of the above procedure we obtained that at the maximum of the measured triple-coincidence distribution the contribution of the accidental coincidences was about $9 \%$.

\section{THEORY}

\section{A. Classical trajectory Monte Carlo calculations}

The CTMC method [37] is based on the solution of the classical mechanical equations of motion of the particles participating in the collision. We applied the three-dimensional, nonrelativistic, four-body version of the theory for the interpretation of the present experimental results. The $\mathrm{He}^{-}$ projectile ion was considered consisting of three particles: two loosely bound electrons around the partially screened core. The He target atom was taken as a single particle, interacting with the other three particles through a completely screened Coulomb force. We made the calculations for the inverse collision system; i.e., we considered the double ionization of the $\mathrm{He}^{-}$ion as target (T) by the impact of the neutral helium atom as projectile $(\mathrm{P})$. The final results were obtained by Galilean transformation of the calculated momenta of the particles.

In the classical treatment of the collisions involving two interacting electrons one is faced with the difficulty caused by the instability of the classical multielectron atoms. Since classically the energy exchange between electrons is not limited, after a time one of the electrons gains enough energy to escape the atom, i.e., autoionization occurs. In quantum mechanics the energy exchange is limited by the Heisenberg uncertainty principle, acting as a repulsing potential in the vicinity of the nucleus. In the classical theoretical models several methods have been proposed to prevent the autoionization (for a review see, e.g., [38]). In the present paper we stabilized the $\mathrm{He}^{-}$ ion by replacing the Coulomb potential for the $e$-T and $e$-e interactions with a soft potential defined as [39]

$$
V_{i j}=\frac{Z_{i} Z_{j}}{\sqrt{r_{i j}^{2}+c_{\alpha \beta}^{2}}} .
$$

Here $c_{\alpha \beta}$ is generally different for the $e$-T and $e-e$ interaction.

The completely screened Coulomb potential around the He projectile was approximated by the model potential developed by Green $e t$ al. [40] on the basis of Hartree-Fock calculations. For an atom it has the following general form:

$$
V(r)=\frac{Z-(N-1)[1-\Omega(r)]}{r},
$$

where $N$ is the number of the electrons in the atom (ion), $Z$ is the nuclear charge, and

$$
\Omega(r)=\{(\eta / \xi)[\exp (\xi r)-1]+1\}^{-1} .
$$

For the neutral He atom the potential was evaluated using $Z=$ $2, N=3, \eta=1.067$, and $\xi=1.188$ [41].

Considering the choice of the initial position and momentum coordinates of the electrons in a two-electron atom (ion), 
unlike the one-electron case [42], there is no unambiguous procedure for the sampling of the microcanonical assembly. We applied a similar trial and error method as that proposed by Cohen [38]. In the first step of our procedure we consider the two electrons independently. We choose the magnitude of their position and momentum randomly in a given interval with uniform distribution. We determine their angular coordinates also randomly in the same way as prescribed in the one-electron case [42]. Then we determine the total energy of the two electrons including their interaction. The sampling was accepted if the difference between the calculated energy and the binding energy ( -0.178 a.u.; see above) was within $1 \%$.

The $c_{\alpha \beta}$ parameters in Eq. (1) were not known for the $\mathrm{He}^{-}$ ion. We varied their values until the stability of the ion was achieved. In the optimization procedure we had to change also the charge of the partially screened core $Z_{\mathrm{T}}$, because the ion turned out to be unstable for $Z_{\mathrm{T}}=1$. Stability was achieved at somewhat larger value, $Z_{\mathrm{T}}=1.3$. For $c_{e \mathrm{~T}}$ and $c_{e e}$ we obtained the values 8.296 and 0.05 a.u., respectively. The stability of $\mathrm{He}^{-}$was checked by performing CTMC calculations for a large number of collision events in a way that we turned off all the interactions with the projectile. For $10^{4}$ collisions we did not observe autoionization.

To prove the existence of the two-electron cusp, the actual calculations had to be made under extreme conditions. Since the cusp is formed by the low-energy electrons emitted in the projectile-centered reference frame, the main requirement was the reliable treatment of the emission of the low-energy electron pairs following the double ionization of $\mathrm{He}^{-}$. The minimum electron energy needed to be determined can be estimated from the experimental energy resolution $\Delta E=$ $0.67 \mathrm{eV}$ at the cusp maximum, $E_{0}=40.8 \mathrm{eV}$. One obtains from velocity transformation that the range $\Delta E$ around $E_{0}$ is built up by electrons emitted in the projectile frame with energies $<2.7 \mathrm{meV}$.

The integration of Newton's equations of motion was started at a distance of $10^{4}$ a.u. between the target and projectile. Such large distance is explained by the time needed to reach the equilibrium in the distribution of the phase-space coordinates of the two electrons in $\mathrm{He}^{-}$well before the collision. The reaction channels (excitation and single and double ionization) in the outgoing phase were checked at even larger distance, at $5 \times 10^{4}$ a.u. This choice was justified by the enhanced probability of the transitions between the reactions channels for such extremely small excitation and emission energies as those discussed above. In the case of double ionization the integration was continued logarithmically until $10^{12}$-a.u. separation.

The calculations were very time consuming due to the small number of the double-ionization events leading to forward electron emission of an electron pair. We followed the history of altogether $10^{9}$ collisions.

In Sec. II we presented our procedure applied for the estimation of the acceptance (half) angle of the electron detection. Using the present CTMC results we could also estimate $\Delta \vartheta_{1 / 2}$. For this purpose we considered the measured electron energy spectrum belonging to the $e-\mathrm{He}^{+}$doublecoincidence events. This spectrum is inclusive in the sense that only one of the electrons is detected at $\vartheta \approx 0^{\circ}$; the

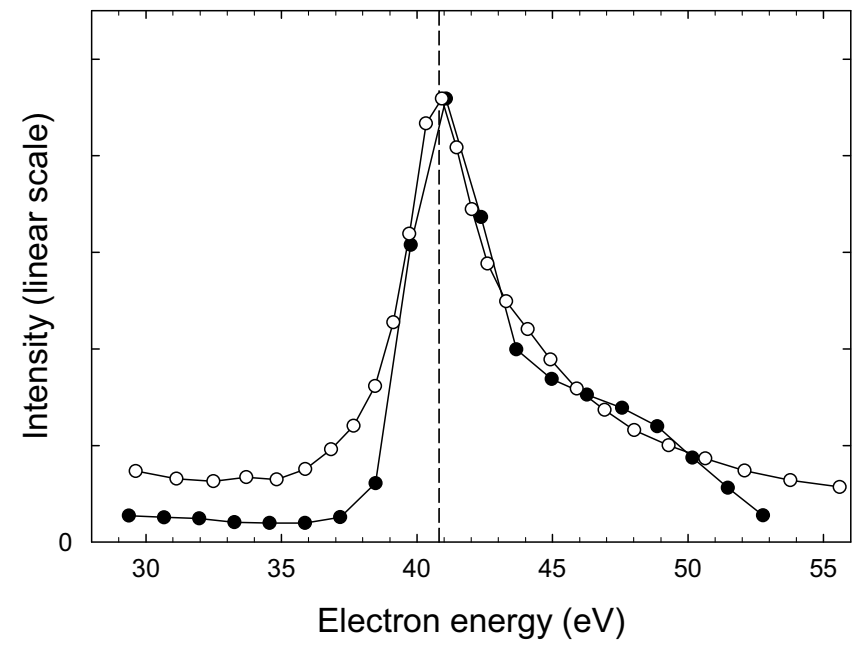

FIG. 2. Single electron cusp spectra following DEL of $\mathrm{He}^{-}$. The open circles denote the distribution measured by detecting double coincidences between the electrons and the outgoing $\mathrm{He}^{+}$ions. The full circles represent the result of the CTMC calculations obtained for acceptance (half) angle $\Delta \vartheta_{1 / 2}=1.2^{\circ}$. The peaks are normalized at their maxima. Here and in the following figures the dashed line shows the expected position of the cusp.

other electron is emitted with an unknown momentum into the full solid angle. This means that the double-coincidence spectrum is less influenced by the electron correlation effects, and thereby the shape of the DEL cusp, particularly its width, is expected to depend mainly on $\Delta \vartheta_{1 / 2}$, as it is known from the single electron cusp studies [43]. Assuming that the same holds for our CTMC model, from the calculated trajectories we generated the corresponding inclusive DEL spectrum for a series of $\Delta \vartheta_{1 / 2}$ values, and compared them with the measured one. The best agreement was found at $\Delta \vartheta_{1 / 2}=1.2^{\circ}$ (see Fig. 2), confirming the result presented in Sec. II.

In the knowledge of the energy and angular resolution of the electron detection the minimum energy of the threshold electrons in the projectile frame can be estimated. In principle, the energy resolution of $0.6 \mathrm{eV}$ at the center of the cusp allows one to discriminate electrons emitted from the projectile with energy as low as $0.5 \mathrm{meV}$. However, according to a simple calculation, no information can be obtained about the forward and backward angular correlation of such extremely lowenergy electrons because of the relatively large acceptance angle $\Delta \vartheta_{1 / 2}$. At this angular resolution the forward and backward emission $\left(\vartheta_{1 / 2}^{\prime} \geqslant \pi / 2\right)$ will be observable in the laboratory energy spectrum only for threshold energies $\geqslant 12 \mathrm{meV}$. The corresponding range in the laboratory spectrum is \pm $1.4 \mathrm{eV}$ around the center of the cusp. Within this range the information about the angular correlation is limited.

\section{B. Monte Carlo simulation based on Wannier's theory}

As it is discussed in Sec. I, the two-electron cusp can most likely be interpreted as a result of formation of a correlated two-electron Wannier state during the collision. The purpose of the Monte Carlo simulation was to obtain information about how far the experimental data reflect the properties of such a state. In our procedure we randomly create individual 
trajectories for the two electrons with the theoretically predicted energy and angular distribution, and track the path of the electrons until they reach the detectors. Using this method, the experimental conditions of the electron detection can easily be taken into account.

Wannier's theory predicts (e.g., [13]) that the angular correlation of the electron pair is characterized by the Gaussian distribution

$$
\frac{d^{4} \sigma}{d E_{1}^{\prime} d \Omega_{1}^{\prime} d E_{2}^{\prime} d \Omega_{2}^{\prime}} \propto \exp \left[-\frac{1}{2 w^{2}}\left(\theta_{12}^{\prime}-\pi\right)^{2}\right],
$$

where $d^{4} \sigma / d E_{1}^{\prime} d \Omega_{1}^{\prime} d E_{2}^{\prime} d \Omega_{2}^{\prime}$ is the fourfold differential cross section (FDCS) for the two-electron emission differential in the electron energies $E_{1}^{\prime}, E_{2}^{\prime}$ and solid angles $\Omega_{1}^{\prime}, \Omega_{2}^{\prime} . w$ is the width of the Gaussian, and $\theta_{12}^{\prime}$ is the angle between the velocity vectors of the electrons. Primed quantities are used for the description of the electron emission in the projectile frame. The FWHM (full width at half maximum) of the distribution depends on the total energy, $E_{t}^{\prime}=E_{1}^{\prime}+E_{2}^{\prime}$; it is a slowly increasing function of $E_{t}^{\prime}$ :

$$
\mathrm{FWHM}=2 \sqrt{2 \ln 2} w=\alpha E_{t}^{\prime 1 / 4}
$$

The simulation starts with a random creation of electron pairs in the projectile frame with the angular correlation expressed by Eq. (4). We assume that at the time moment when the simulation starts the collision fragments have already separated from each other to asymptotically large distances; i.e., they fly freely without any interaction between them. We assume, furthermore, that the electron pairs are emitted isotropically. In Eq. (5) the value of $\alpha$ was derived from the angular correlation obtained by Barrachina et al. [19] considering the quantum-mechanical version of Wannier's theory:

$$
\begin{aligned}
& \frac{d^{4} \sigma}{d E_{1}^{\prime} d \Omega_{1}^{\prime} d E_{2}^{\prime} d \Omega_{2}^{\prime}} \\
& \propto \exp \left[-\frac{1}{16} \sqrt{\frac{\left(9-4 Z_{\mathrm{T}}\right)\left(4 Z_{\mathrm{T}}-1\right)}{E}}\left(\theta_{12}^{\prime}-\pi\right)^{2}\right] .
\end{aligned}
$$

For $Z_{\mathrm{T}}=1$ and 1.3 one obtains $\alpha=3.38$ and 3.33 , respectively. We note that $\alpha=3.0$ was obtained theoretically by Bartlett and Stelbovics [17] for electron-impact ionization of hydrogen, and was verified experimentally by Williams et al. [11].

For electron-impact ionization the total electron energy $E_{t}^{\prime}$ in Eq. (5) is the excess energy above the ionization threshold which is uniquely determined by the impact energy. In ionatom collision $E_{t}^{\prime}$ has a continuous spectrum; its value depends on the amount of the transferred energy. We may assume that for small excitations above the threshold the cross section for the two-electron emission differential in $E_{t}^{\prime}$ can be expressed by a linear function:

$$
\frac{d \sigma}{d E_{t}^{\prime}} \sim c_{0}+c_{1} E_{t}^{\prime} .
$$

This is justified by the small energy of the electrons contributing to the cusp in the projectile-centered reference system. The laboratory electron energy range of our measurements is $28-56 \mathrm{eV}$. In the projectile system this corresponds to forward and backward emission with only maximum 1-eV energy. The coefficients $c_{0}$ and $c_{1}$ are free parameters of the model. In the experiment we measured relative cross sections, therefore only the ratio of the two coefficients has to be considered.

As a further justification of Eq. (7) let us consider the cross section integrated over $E_{t}^{\prime}$ :

$$
\sigma \sim c_{0} E_{t}^{\prime}+c_{1} E_{t}^{\prime 2} / 2
$$

At very small values of $E_{t}^{\prime}$ the quadratic term is negligible, i.e., $\sigma \sim E_{t}^{\prime}$. This is very close to the prediction of the Wannier theory, $\sigma \sim E^{1.127}$.

It is important to note that the fact that the differential cross section in Eq. (7) does not vanish at $E_{t}^{\prime}=0$ automatically leads to the appearance of the cusp. To show this, let us consider the FDCS of the two-electron ejection which is differential in the electron velocities, $d^{4} \sigma / d \mathbf{v}_{1} d \mathbf{v}_{2}$. This quantity is invariant with respect to the reference frame transformation. Accordingly, we have the following relationship between the FDCS in the laboratory and projectile frame:

$$
\frac{d^{4} \sigma}{v_{1}^{2} d v_{1} d \Omega_{1} v_{2}^{2} d v_{2} d \Omega_{2}}=\frac{d^{4} \sigma^{\prime}}{v_{1}^{\prime 2} d v_{1}^{\prime} d \Omega_{1}^{\prime} v_{2}^{\prime 2} d v_{2}^{\prime} d \Omega_{2}^{\prime}} .
$$

Since $v_{i}^{2} d v_{i}$ can be expressed as $v_{i} d E_{i}$ (also for the primed quantities), we obtain

$$
\frac{d^{4} \sigma}{d E_{1} d \Omega_{1} d E_{2} d \Omega_{2}}=\frac{v_{1} v_{2}}{v_{1}^{\prime} v_{2}^{\prime}} \frac{d^{4} \sigma^{\prime}}{d E_{1}^{\prime} d \Omega_{1}^{\prime} d E_{2}^{\prime} d \Omega_{2}^{\prime}} .
$$

It can be seen that if the FDCS in the projectile frame does not vanish at $E_{1}^{\prime}=E_{2}^{\prime}=0$ then the FDCS in the laboratory frame is divergent for the ejection of both electrons; i.e., the two-electron cusp appears. The $c_{0} \neq 0$ in Eq. (7) together with other assumptions of our model ensures the nonvanishing FDCS in the projectile frame, and thereby provides the possibility for the formation of the two-electron cusp.

In the simulation the electron energies $E_{1}^{\prime}$ and $E_{2}^{\prime}$ are obtained as follows. First we select the value of the total electron energy $E_{t}^{\prime}$ randomly with the distribution defined by Eq. (7). Then we share $E_{t}^{\prime}$ between the two electrons assuming uniform distribution. The angular correlation given by Eqs. (4) and (5) and the uniform energy share are the most important predictions of the Wannier threshold theories (see, e.g., [13]). The latter prediction has been verified experimentally [5,9].

Using the obtained electron energies and emission angles, we determine the velocity vectors of the electrons. The energies $E_{1}$ and $E_{2}$ in the laboratory frame are calculated by Galilean transformation of the velocities. All the characteristics of the time-of-flight measurement (acceptance angle, time resolution, finite projectile beam size, extended gas target) are taken into account in the simulation.

\section{RESULTS AND DISCUSSION}

From the measured triple-coincidence yields we determined relative FDCSs by subtracting the random coincidences and applying corrections for the detection efficiency of the two electron branches. The result is displayed in Fig. 3. In panel (a) the 3D representation of FDCS clearly shows a peak at the expected cusp position for both electrons, giving evidence for existence of the two-electron cusp in DEL of 


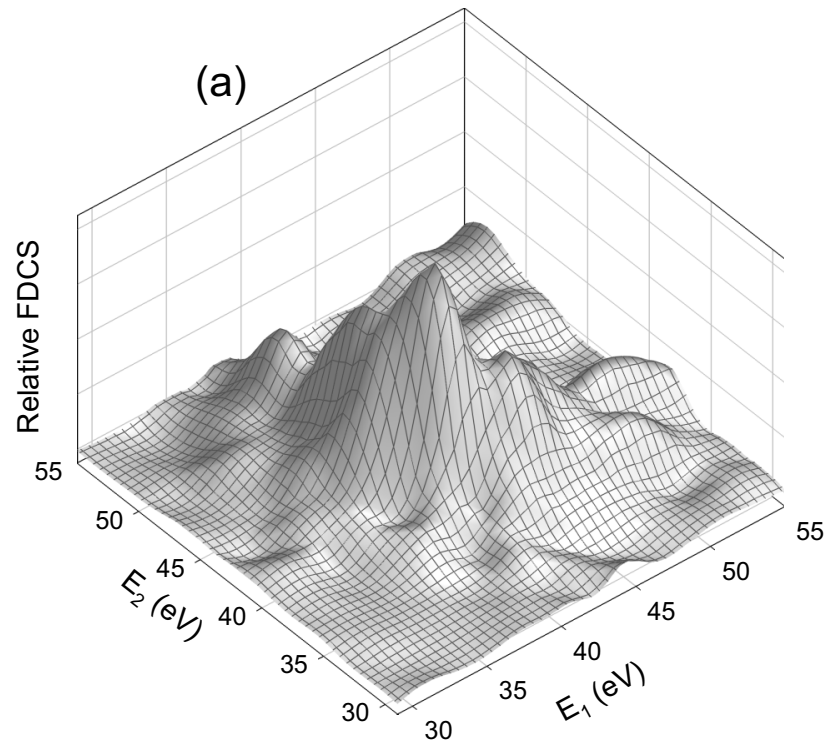

(b)

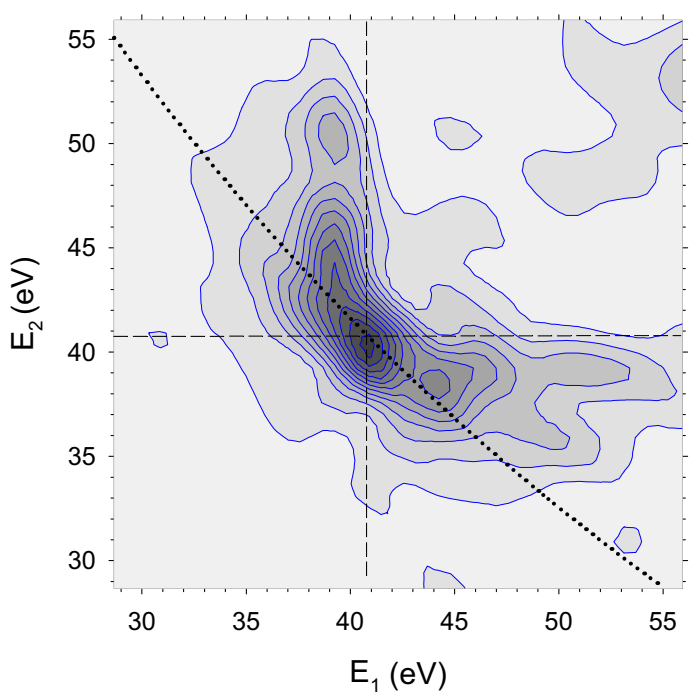

FIG. 3. The measured relative FDCS for the two-electron emission in DEL of $\mathrm{He}^{-}$as a function of the energies of the electron pairs. In panels (a) and (b) the scale of the FDCS is linear. For the explanation of the dotted line in panel (b) see the main text.

$\mathrm{He}^{-}$. According to the contour plot of the data in panel (b), the emission of the electron pairs is highly anticorrelated: An electron ejected with an energy higher (lower) than the cusp energy $\left(E_{0}=40.8 \mathrm{eV}\right)$ is most likely accompanied by an electron ejected with an energy lower (higher) than $E_{0}$. In the projectile frame this corresponds to back-to-back emission.

The dotted line in the contour plot shows the correlation that could be observed in the limit of infinitely sharp angular correlation of $180^{\circ}$, for infinitely good angular and energy resolution, and for equal energy share between the electrons in the projectile frame. Its equation

$$
E_{2}=4 E_{0}+E_{1}-4 \sqrt{E_{0} E_{1}}
$$

is obtained by solving the system of equations $v_{1}=v_{0}+v^{\prime}$ and $v_{2}=v_{0}-v^{\prime}$, where $v_{1}$ and $v_{2}$ are the laboratory-frame

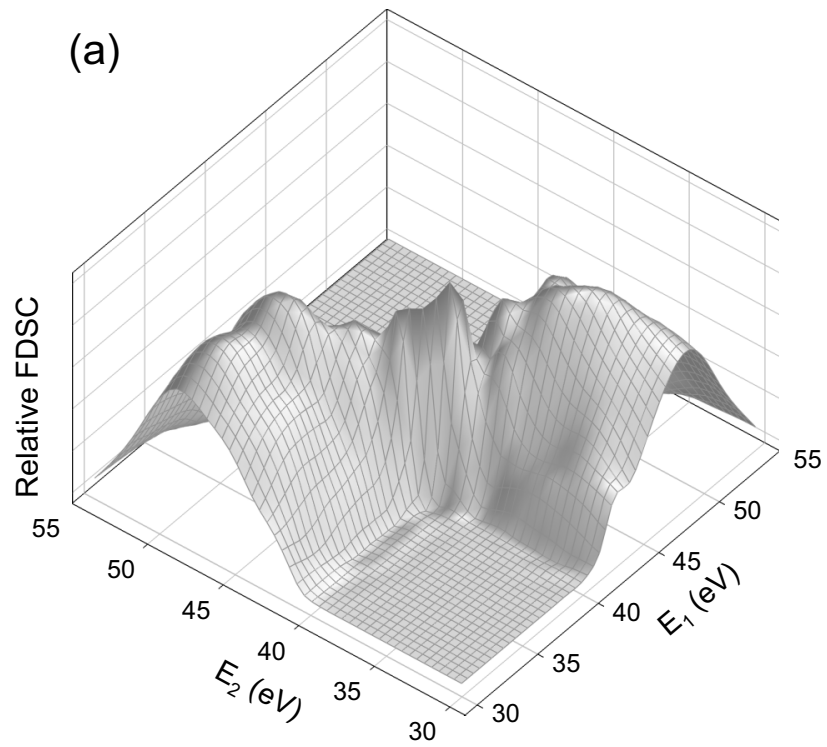

(b)

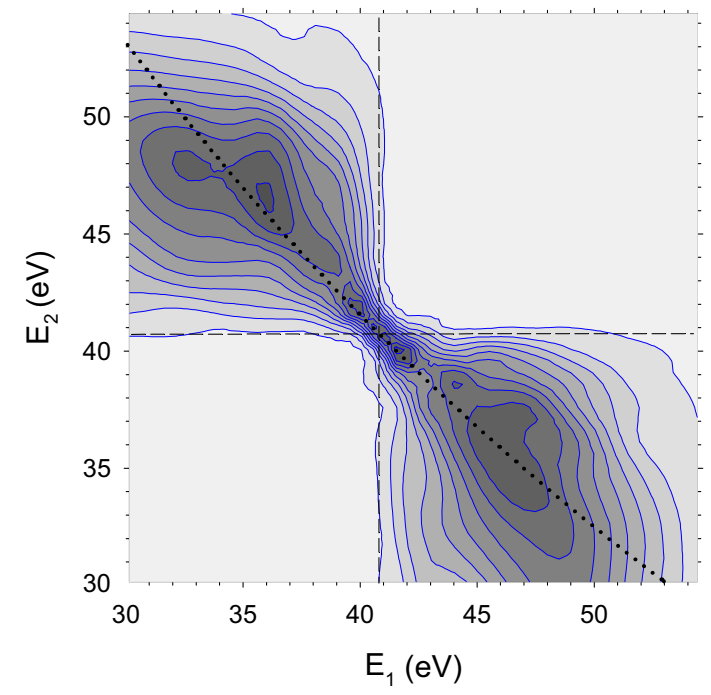

FIG. 4. The relative FDCS for the two-electron emission in DEL of $\mathrm{He}^{-}$obtained by the present CTMC calculations.

velocities, and $\pm v^{\prime}$ are the projectile-frame velocities of the electrons.

The distribution of the energies of the electron pairs forms a ridge. In the vicinity of the peak the ridge follows well the line given by Eq. (11). Farther from the peak the ridge increasingly deviates from this line towards larger energies. This is in contrast to the correlation pattern observed in our first experiment on the two-electron cusp [27], which had a butterfly shape and showed almost a mirror symmetry with respect to the line of the complete anticorrelation.

Figure 4 shows the results of the CTMC calculations. According to panel (a), the existence of the two-electron cusp is uncertain. Although the distribution has a peak at the expected cusp position, it is not well pronounced; one cannot exclude that it is only a statistical fluctuation of the intense, long ridge dominating the distribution. At the same time, as is seen in panel (b), CTMC predicts highly anticorrelated emission of 


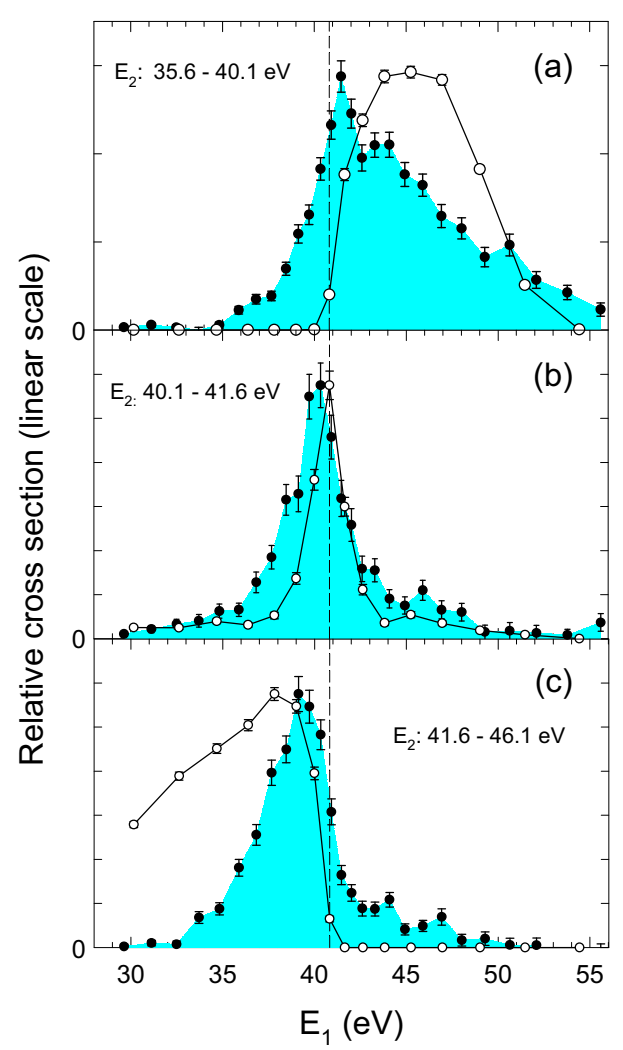

FIG. 5. Energy spectra of the electron $e_{1}$ emitted from DEL of $\mathrm{He}^{-}$with the condition that the energy of $e_{2}$ lies in the interval (a) $35.6-40.1 \mathrm{eV}$, (b) $40.1-41.6 \mathrm{eV}$ and (c) $41.6-46.1 \mathrm{eV}$. The full circles denote the experimental data. The open circles represent the CTMC calculations. The experimental and theoretical data are normalized at the peak maxima.

the two electrons, in accordance with the experiment. However, in this case the ridge of the calculated FDCSs follows the line given by Eq. (11) through the entire considered energy range. Furthermore, the distribution of FDCSs shows a mirror symmetry with respect to the latter line, in strong disagreement with the experiment.

The above mirror symmetry of the calculated FDCSs can be well understood physically. In the final state of the collision the only charged particles are the two electrons and the projectile core. The screened Coulomb field of the receding neutral target atom has practically no effect on the motion of the other three particles. In other words, the Wannier two-electron state is unperturbed, and the motion of the two electrons is symmetric. In this context we mention that in our first study of the two-electron cusp [27] this was not the case. There the two electrons originated from the mutual ionization of the projectile and target, therefore in the final state the motion of the electrons was influenced by the Coulomb field of the receding ionized target, too. This postcollision interaction (PCI) effect resulted in a small deviation of the distribution of FDCSs from the mirror symmetry towards lower energies.

For a detailed comparison between the experiment and theory we considered some specific ranges of FDCS. In Fig. 5 we displayed measured and calculated energy spectra of one of the electrons, $e_{1}$, ejected from DEL of $\mathrm{He}^{-}$with the condition
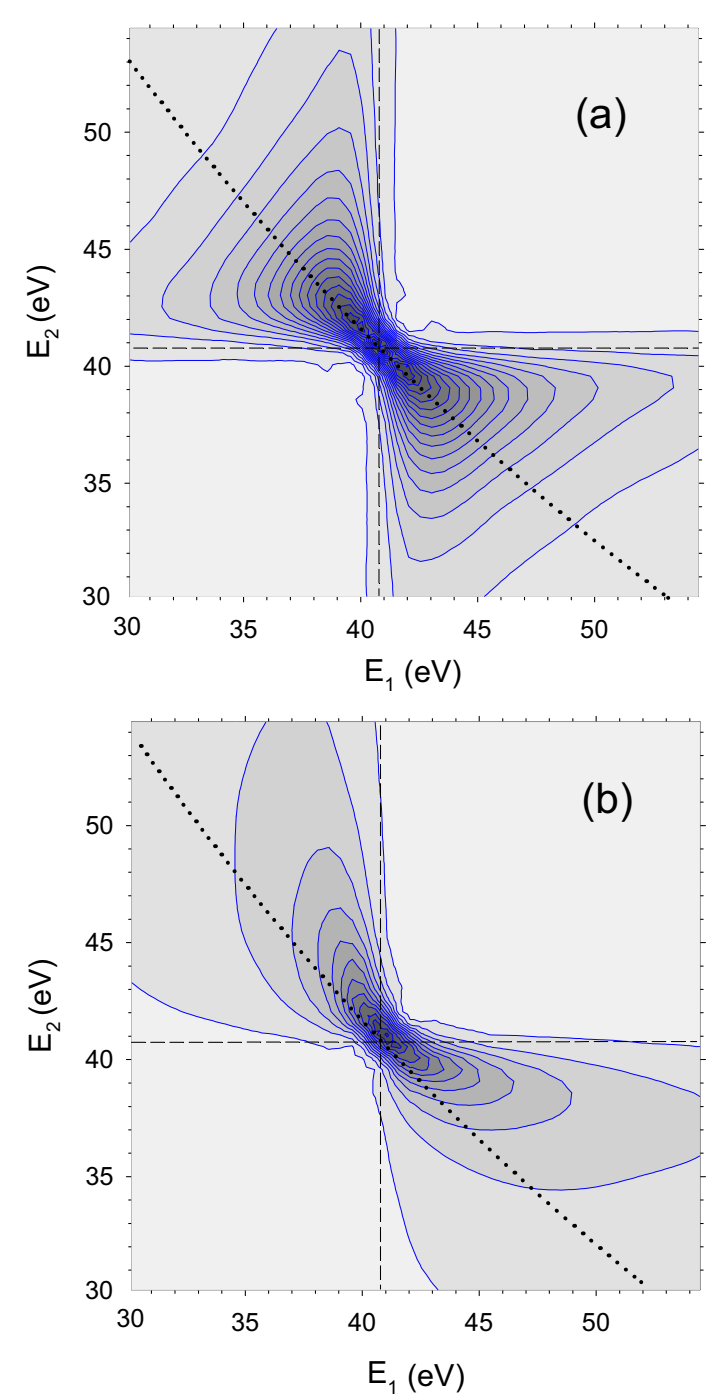

FIG. 6. Results of the Monte Carlo simulations. Panel (a) represents the correlation pattern expected from Wannier's theory. Panel (b) shows the distribution perturbed by postcollision interaction.

that the energy of the other electron, $e_{2}$, lies in a narrow range. We compare only the shapes of the spectra, therefore we normalized the calculated spectra to the experimental ones at the peak maxima. According to the figure, CTMC provides only a qualitative description of the experimental data: For cases displayed in Figs. 5(a) and 5(c) it predicts larger shifts and broadening of the peak than those observed in the experiment. For the case shown in Fig. 5(b) $\left(E_{2} \approx E_{0}\right)$ the calculated peak is considerably narrower than the measured one.

In the following we discuss the results obtained by our Monte Carlo simulations (see Sec. III B). Figure 6(a) shows the contour plot of the distribution of the energies of the electron pairs created according to Wannier's theory, i.e., assuming the angular correlation given by Eqs. (4) and (5) and uniform share of the total excitation energy. In Eq. (5) $\alpha=3.38$ was used, and the experimentally observed intensity of the two-electron cusp was reproduced by taking $c_{0} / c_{1}=$ $1.67 \times 10^{-3}$ in Eq. (7). From comparison of Figs. 3(b) and 6 (a) one can conclude that the prediction of Wannier's theory 
is in complete disagreement with the experiment. At the same time, the agreement between the simulation and the CTMC result shown in Fig. 4(b) is somewhat better.

There are two striking differences between the measured and simulated correlation patterns.

(i) The simulated distribution has a butterfly shape which is due to the increasing width of the angular correlation with increasing total excitation energy $E_{t}^{\prime}$ according to Eq. (5). In contrast to this, the measured distribution is more concentrated along a ridge, indicating almost a constant width of the angular correlation.

(ii) The simulated distribution shows a mirror symmetry with respect to the line given by Eq. (11). The measured distribution does not show this symmetry; the ridge strongly curves from the line of symmetry towards larger energies.

We attempted to reproduce the experimental correlation pattern by suitable choice of the parameters of the Monte Carlo simulation. The model allowed us to reconstruct only the main features of the experimental distribution. The best result is shown in Fig. 6(b). It was obtained by the following parameters. We used a constant width of the angular correlation, $\mathrm{FWHM}=\pi / 8$. A two-electron cusp comparable with the measured one was obtained with a modified function for $d \sigma / d E_{t}^{\prime}$,

$$
\frac{d \sigma}{d E_{t}^{\prime}} \sim c_{0}+c_{1} E_{t}^{\prime 1 / 2}
$$

with the choice $c_{0} / c_{1}=6.67 \times 10^{-3}$. The deviation of the distribution from the mirror symmetry was accounted for by increasing the velocity of both electrons in the projectile frame by $\Delta v^{\prime}=3 v^{\prime 3 / 2}$.

On the basis of the modified Monte Carlo simulation we tried to understand the physical process leading to the twoelectron cusp in DEL of $\mathrm{He}^{-}$. It is clear that the main mechanism is not the formation of the Wannier-type correlated two-electron state. This is excluded by the constant width of the angular correlation. The increased velocities of the electrons in the projectile frame indicate the presence of a PCI effect. As it was mentioned above, we found evidence of the role of PCI in the formation of the two-electron cusp in our previous study of the process [44]. In that case PCI was due to the Coulomb field of the ionized target which attracted the electrons backward. In the simulation this was expressed by decreasing the velocity of the electrons in the projectile frame; i.e., the sign of $\Delta v^{\prime}$ was negative.

The positive $\Delta v^{\prime}$ found in the present investigations means a repulsive PCI. The most obvious candidate for such an interaction is that exerted by a third electron. As we mentioned in Sec. I, the coincidence conditions did not exclude the simultaneous ionization of the target. However, the probability of the process is very small due to the strongly bound electrons in He. A repulsive force may also arise as a result of the polarization of the target by the Coulomb field of the projectile core. In principle, the dipolar force has long range [24], and thereby it may perturb the motion of the two electrons. However, the collision-induced dipole momentum in the $\mathrm{He}$ atom is not permanent $[23,24]$, and therefore its effect is uncertain at asymptotically large separation between the collision partners.

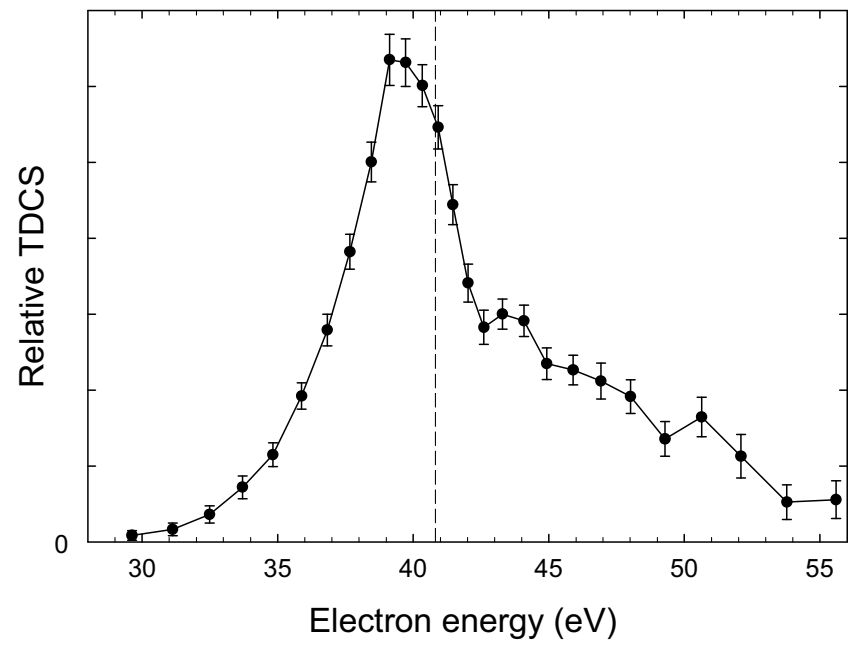

FIG. 7. Relative triply differential cross section obtained by integration of the measured relative FDCS over the energy of one of the two electrons ejected in DEL of $\mathrm{He}^{-}$.

A closer look at the measured relative FDCS shown in Fig. 3 reveals some structure: A shoulder is seen on both sides of the peak at electron energies at $E_{1(2)}=39 \mathrm{eV}$ and $E_{2(1)}=43 \mathrm{eV}$. The structure is very uncertain due to the poor statistics. To confirm its existence, we integrated the FDCS over the energy of one of the two electrons. The obtained spectrum (relative triply differential cross section, TDCS) is plotted in Fig. 7. It shows a cusp that is shifted to lower energies. On the high-energy side of the peak the shoulder (or peak) is clearly visible at $43-\mathrm{eV}$ electron energy. We note that the shift of the cusp can probably be explained by the contribution of the unresolved low-energy shoulder at $39 \mathrm{eV}$. If it is so, the two shoulders are located symmetrically on the low- and high-energy side of the cusp. The shape of the spectrum suggests two mechanisms of the two-electron emission: Direct double ionization of $\mathrm{He}^{-}$leading to the cusp and a resonant process resulting in the structure superimposed on the cusp. Resonantly two low-energy electrons may be emitted as a result of single electron detachment of $\mathrm{He}^{-}$and subsequent shakeoff of the neutralized He. Since the shakeoff is delayed with respect to the primary process, the question arises how far the motions of the electrons are anticorrelated. Another possible resonant process is the formation of a triply excited state of $\mathrm{He}^{-}$(for a review see, e.g., [45]) followed by simultaneous double-Auger (DA) decay [46]. In this case strong angular correlation is expected between the electrons [47]. For $\mathrm{He}^{-}$the DA process was observed for the triply excited $2 s 2 p^{2}{ }^{4} P$ state [48]. Since this state decays to the $1 s$ state of $\mathrm{He}^{+}$, the energies of the emitted electrons are too large to be observed in our experiment. Small DA energies are expected only from the decay of highly excited $n n^{\prime} l^{\prime} n^{\prime \prime} l^{\prime \prime}$ states, where $n, n^{\prime}$, and $n^{\prime \prime}$ have large values. Lacking any information about such states and their decay properties, we cannot estimate the contribution of the DA process to the relative FDCS measured in the present paper. We note that for the inclusion of the resonant processes their interference with the direct process has to be also considered. 


\section{CONCLUSIONS}

We carried out a triple-coincidence experiment for the study of the two-electron cusp formed in DEL of $\mathrm{He}^{-}$induced by collisions with $\mathrm{He}$ atoms at $300-\mathrm{keV}$ impact energy. We established the existence of the cusp in the energy distribution of the forward emitted electron pairs. We found that the motions of the electrons contributing to the cusp are strongly anticorrelated. Our Monte Carlo simulations revealed that the observed cusp cannot be understood in terms of Wannier's theory. The most striking deviation from the theory is that the width of the angular correlation of the electron pairs does not increase with the total excitation energy. Furthermore, the energy distribution of the electron pairs does not show mirror symmetry with respect to the line of the complete anticorrelation. We considered as a reason for the latter deviation PCI exerted by a third emitted electron or by the polarized target on the electron pairs.

We compared our experimental data also with the results of four-body CTMC calculations carried out applying the
soft-Coulomb potential approximation for the inclusion of the electron-electron interaction. The calculations do not prove unambiguously the existence of the two-electron cusp. The obtained energy correlation pattern of the electron pairs is in disagreement with the measured data; rather, it is in accordance with the expectation of Wannier's theory.

A further result of the present investigations is the observation of a structure in the measured relative FDCS. The most probable explanation of the structure is the formation of a high-lying, triply excited state of $\mathrm{He}^{-}$followed by simultaneous double-Auger decay. This observation calls for a new way of exploring the triply excited states of $\mathrm{He}^{-}$by means of zero-degree electron spectroscopy.

\section{ACKNOWLEDGMENTS}

This work was supported by the National Scientific Research Foundation (Hungarian Scientific Research Fund Grants No. K109440 and No. K128621) as well as the National Information Infrastructure Program.
[1] E. P. Wigner, Phys. Rev. 73, 1002 (1948).

[2] H. R. Sadeghpour, J. L. Bohn, M. J. Cavagnero, B. D. Esry, I. I. Fabrikant, J. H. Macek, and A. R. P. Rau, J. Phys. B 33, R93 (2000).

[3] H. Hotop, M.-W. Ruf, and I. I. Fabrikant, Phys. Scr. T110, 22 (2004).

[4] G. H. Wannier, Phys. Rev. 90, 817 (1953).

[5] S. Cvejanović and F. H. Read, J. Phys. B 7, 1841 (1974).

[6] J. B. Donahue, P. A. M. Gram, M. V. Hynes, R. W. Hamm, C. A. Frost, H. C. Bryant, K. B. Butterfield, D. A. Clark, and W. W. Smith, Phys. Rev. Lett. 48, 1538 (1982).

[7] P. Selles, J. Mazeau, and A. Huetz, J. Phys. B 23, 2613 (1990).

[8] J. Mazeau, P. Selles, D. Waymel, and A. Huetz, Phys. Rev. Lett. 67, 820 (1991).

[9] P. Lablanquie, K. Ito, P. Morin, I. Nenner, and J. H. D. Eland, Z. Phys. D 16, 77 (1990).

[10] P. Lablanquie, J. Mazeau, L. Andric, P. Selles, and A. Huetz, Phys. Rev. Lett. 74, 2192 (1995).

[11] J. F. Williams, P. L. Bartlett, and A. T. Stelbovics, Phys. Rev. Lett. 96, 123201 (2006).

[12] R. Peterkop, J. Phys. B 4, 513 (1971).

[13] A. R. P. Rau, J. Phys. B 9, L283 (1976).

[14] F. H. Read and S. Cvejanović, J. Phys. B 21, L371 (1988).

[15] A. Huetz, P. Selles, D. Waymel, and J. Mazeau, J. Phys. B 24, 1917 (1991).

[16] L. Malegat, P. Selles, P. Lablanquie, J. Mazeau, and A. Huetz, J. Phys. B 30, 263 (1997).

[17] P. L. Bartlett and A. T. Stelbovics, Phys. Rev. Lett. 93, 233201 (2004).

[18] J. S. Briggs and V. Schmidt, J. Phys. B 33, R1 (2000).

[19] R. O. Barrachina, L. Gulyás, and L. Sarkadi, Nucl. Instrum. Methods Phys. Res., Sect. B 369, 98 (2016).

[20] Á. Kövér, L. Sarkadi, J. Pálinkás, D. Berényi, G. Szabó, T. Vajnai, O. Heil, K. O. Groeneveld, J. Gibbons, and I. A. Sellin, J. Phys. B 22, 1595 (1989).
[21] L. Sarkadi, J. Pálinkás, Á. Kövér, D. Berényi, and T. Vajnai, Phys. Rev. Lett. 62, 527 (1989).

[22] F. Penent, J. P. Grouard, J. L. Montmagnon, and R. I. Hall, J. Phys. B 25, 2831 (1992).

[23] L. Víkor and L. Sarkadi, Phys. Rev. A 55, R2519 (1997).

[24] L. Sarkadi, Tókési, and R. O. Barrachina, J. Phys. B 33, 847 (2000).

[25] L. Sarkadi and D. Nagy, Phys. Rev. A 94, 042709 (2016).

[26] W. Schmitt, R. Moshammer, F. S. C. O'Rourke, H. Kollmus, L. Sarkadi, R. Mann, S. Hagmann, R. E. Olson, and J. Ullrich, Phys. Rev. Lett. 81, 4337 (1998).

[27] L. Sarkadi and A. Orbán, Phys. Rev. Lett. 100, 133201 (2008).

[28] L. Gulyás, L. Sarkadi, A. Igarashi, and T. Kirchner, Phys. Rev. A 82, 032705 (2010).

[29] J. Xi and C. F. Fischer, Phys. Rev. A 53, 3169 (1996).

[30] P. Reinhed, A. Orbán, J. Werner, S. Roseń, R. D. Thomas, I. Kashperka, H. A. B. Johansson, D. Misra, L. Brännholm, M. Björkhage, H. Cederquist, and H. T. Schmidt, Phys. Rev. Lett. 103, 213002 (2009).

[31] P. A. Závodszky, L. Sarkadi, L. Víkor, and J. Pálinkás, Phys. Rev. A 50, R899(R) (1994).

[32] A. Báder, L. Sarkadi, L. Víkor, M. Kuzel, P. A. Závodszky, T. Jalowy, K. O. Groeneveld, P. A. Macri, and R. O. Barrachina, Phys. Rev. A 55, R14 (1997).

[33] L. Sarkadi and A. Orbán, Meas. Sci. Technol. 17, 84 (2006).

[34] A. Báder, L. Sarkadi, Gy. Hegyesi, L. Víkor, and J. Pálinkás, Meas. Sci. Technol. 6, 959 (1995).

[35] L. Víkor, L. Sarkadi, K. Tőkési, D. Varga, F. Penent, and J. Pálinkás, Nucl. Instrum. Methods Phys. Res., Sect. B 114, 164 (1996).

[36] L. Sarkadi and R. O. Barrachina, Phys. Rev. A 71, 062712 (2005).

[37] R. Abrines and I. C. Percival, Proc. Phys. Soc. London 88, 861 (1966).

[38] J. S. Cohen, Phys. Rev. A 54, 573 (1996).

[39] Q. Su and J. H. Eberly, Phys. Rev. A 44, 5997 (1991). 
[40] A. E. S. Green, D. L. Sellin, and A. S. Zachor, Phys. Rev. 184, 1 (1969).

[41] R. H. Garvey, C. H. Jackman, and A. E. S. Green, Phys. Rev. A 12, 1144 (1975).

[42] C. O. Reinhold and R. E. Olson, Phys. Rev. A 39, 3861 (1989).

[43] Á. Kövér, G. Szabó, D. Berényi, L. Gulyás, I. Cserny, K. O. Groeneveld, D. Hofmann, P. Koschar, and M. Burkhard, J. Phys. B 19, 1187 (1986).
[44] L. Sarkadi and A. Orbán, Nucl. Instrum. Methods Phys. Res., Sect. B 267, 270 (2009).

[45] T. Andersen, Phys. Rep. 394, 157 (2004).

[46] T. A. Carlson and M. O. Krause, Phys. Rev. Lett. 14, 390 (1965).

[47] M. Ya. Amusia, I. S. Lee, and V. A. Kilin, Phys. Rev. A 45, 4576 (1992).

[48] R. C. Bilodeau, J. D. Bozek, A. Aguilar, G. D. Ackerman, G. Turri, and N. Berrah, Phys. Rev. Lett. 93, 193001 (2004). 